\title{
On Comparison of the Estimators of the Hurst Index of the Solutions of Stochastic Differential Equations Driven by the Fractional Brownian Motion
}

\author{
Kęstutis KUBILIUS ${ }^{1 *}$, Dmitrij MELICHOV ${ }^{2}$ \\ ${ }^{1}$ Vilnius University Institute of Mathematics and Informatics \\ Akademijos 4, LT-08663 Vilnius, Lithuania \\ ${ }^{2}$ Vilnius Gediminas Technical University \\ Saulètekio 11, LT-10223 Vilnius, Lithuania \\ e-mail:kestutis.kubilius@mii.vu.lt
}

Received: August 2010; accepted: November 2010

Abstract. This paper presents a study of the Hurst index estimation in the case of fractional Ornstein-Uhlenbeck and geometric Brownian motion models. The performance of the estimators is studied both with respect to the value of the Hurst index and the length of sample paths.

Keywords: fractional Brownian motion, Hurst index, Ornstein-Uhlenbeck process, geometric Brownian motion.

\section{Introduction}

In this paper, we consider the stochastic differential equation

$$
X_{t}=c+\int_{0}^{t} f\left(X_{s}\right) \mathrm{d} s+\int_{0}^{t} g\left(X_{s}\right) \mathrm{d} B_{s}^{H}, \quad t \in[0 ; 1], c \in \mathbb{R},
$$

where $B_{t}^{H}$ is the fractional Brownian motion (fBm) with the Hurst index $1 / 2<H<1$. $\mathrm{fBm}$ is a continuous-time Gaussian process $\left\{B_{t}^{H}, t \geqslant 0\right\}$ with $B_{0}^{H}=0$ and the covariance function

$$
\mathbf{E}\left[B_{t}^{H} B_{s}^{H}\right]=\frac{1}{2}\left(|t|^{2 H}+|s|^{2 H}-|t-s|^{2 H}\right) .
$$

The Hurst index $H$ determines the correlation between the increments of $\mathrm{fBm}$. If $H<1 / 2$, these increments are negatively correlated, if $H>1 / 2$, the increments are positively correlated, and if $H=1 / 2$, the process is a regular Brownian motion. In

*Supported by the Research Council of Lithuaniam contract No. MIP-66/2010. 
this paper the case $H>1 / 2$ is considered since it allows to model various phenomena possessing the long-range dependance property. The second integral in (1) is that of Riemann-Stieltjes defined pathwise. For $0<\alpha \leqslant 1, \mathcal{C}^{1+\alpha}(\mathbb{R})$ denotes the set of all $\mathcal{C}^{1}$-functions $g: \mathbb{R} \rightarrow \mathbb{R}$ such that

$$
\sup _{x}\left|g^{\prime}(x)\right|+\sup _{x \neq y} \frac{\left|g^{\prime}(x)-g^{\prime}(y)\right|}{|x-y|^{\alpha}}<\infty .
$$

Let $f$ be a Lipschitz function, $g \in \mathcal{C}^{1+\alpha}(\mathbb{R}), 0<\alpha \leqslant 1$. It is known (Lyons, 1994; Dudley, 1999; Kubilius, 2000; Nualart and Răşcanu, 2002) that there exists a unique strong solution of (1).

The fractional Brownian motion and processes based on it have found many applications in fields as diverse as economics and finance, physics, chemistry, medicine and environmental studies. Computer science is not an exception - $\mathrm{fBm}$ has been employed in telecommunication traffic studies (for an overview, see Li, 2010 and the references therein), fractal image compression (Davis, 1996), image object detection (see, i.e., Wenlu and Weixin, 1997) and other studies. Obviously, if some phenomenon can be modeled by $\mathrm{fBm}$ or a process based on it, the estimation of the $\mathrm{fBm}$ 's parameter, the Hurst index $H$, is an important problem.

In 1961, Gladyshev (1963) derived a limit theorem for a statistic based on the first order quadratic variations of $\mathrm{fBm}$. This yielded an estimator of $H$ which was strongly consistent. In 1997, another estimator was introduced by Istas and Lang (1997) which again employed the first order quadratic variations and it was asymptotically normal for $H \in(1 / 2 ; 3 / 4)$. In 2005, Bégyn (2005) considered the second order quadratic variations along general subdivisions for processes with Gaussian increments. A more complete survey about asymptotic behavior of quadratic variations for Gaussian processes can be found in the thesis Bégyn (2006). In 2006, Berzin and León (2008) proposed a CLT for estimators of $H$ and the diffusion function $g$ for several specific cases of (1). In 2008-2010, Kubilius and Melichov $(2008,2009,2010)$ studied the behavior of the first and second order quadratic variations of the pathwise solution of (1) and have shown that the quadratic variation based estimators remain strongly consistent in that case as well.

The goal of this paper is to compare the behavior of the estimators based on quadratic variations with some of the other known estimators, namely the naive and ordinary least squares Gladyshev and $\eta$-summing oscillation estimators, the variogram estimator and the IR estimator. These estimators are described in Section 1.2. Most of them were examined for Gaussian processes. The models chosen for comparison of these estimators were the fractional Ornstein-Uhlenbeck (O-U) and the fractional geometric Brownian motion $(\mathrm{gBm})$. The initial inference about the behavior of these estimators was drawn for the O-U process which is Gaussian, while the $\mathrm{gBm}$ process was used to check how the estimators behave in a non-Gaussian case.

In order to achieve that, a sufficient amount of $\mathrm{fBm}$ sample paths is required. These sample paths were generated using the circulant matrix embedding method, as described in Coeurjolly (2000) and the references therein. Let $n$ denote the length of the sample path. The circulant matrix embedding method uses a fast Fourier transform which 
bypasses matrix computations and therefore is sufficiently fast even for large values of $n$. 100 sample paths of the length $n=2^{14}+1$ were generated for each value of $H \in\{0.55,0.6, \ldots, 0.95\}$ on the unit interval $t \in[0 ; 1]$.

The next step would be to use the generated fBm data to construct sample paths of the considered processes. However, it's not always possible to find and use the explicit solution of (1), therefore this solution needs to be replaced by a time discrete approximation. For a process $X_{t}$, its Milstein approximation at points $t_{k}^{n}, k=1, \ldots, n$ is defined as

$$
X_{k}^{n}=X_{k-1}^{n}+f\left(X_{k-1}^{n}\right) \Delta t_{k}+g\left(X_{k-1}^{n}\right) \Delta B_{k}^{H}+\frac{1}{2} g\left(X_{k-1}^{n}\right) g^{\prime}\left(X_{k-1}^{n}\right)\left(\Delta B_{k}^{H}\right)^{2},
$$

where $g^{\prime}$ denotes the derivative of $g$ and $X_{0}^{n}=c$. The fractional Ornstein-Uhlenbeck $(\mathrm{O}-\mathrm{U})$ and the fractional geometric Brownian motion $(\mathrm{gBm})$ processes are defined as

$$
\begin{aligned}
& \mathrm{d} X_{t}=-\mu X_{t} \mathrm{~d} t+\sigma \mathrm{d} B_{t}^{H}, \quad X_{0}=c, \\
& \mathrm{~d} X_{t}=\mu X_{t} \mathrm{~d} t+\sigma X_{t} \mathrm{~d} B_{t}^{H}, \quad X_{0}=c .
\end{aligned}
$$

The solutions of these equations are, respectively,

$$
X_{t}=e^{-\mu t}\left(c+\sigma \int_{0}^{t} e^{\mu s} \mathrm{~d} B_{s}^{H}\right) \quad \text { and } \quad X_{t}=c \exp \left(\mu t+\sigma B_{t}^{H}\right) .
$$

In fact, for the O-U process the Milstein approximation is reduced to the Euler one due to $g^{\prime}\left(X_{k-1}^{n}\right)=(\sigma)^{\prime}=0$. The constants were chosen as $c=1, \mu=0.5, \sigma=0.7$ in the $\mathrm{O}-\mathrm{U}$ case and $c=1, \mu=0.2, \sigma=0.5$ in the $\mathrm{gBm}$ case. The error introduced by using these approximated sample paths was negligible compared to the errors of the estimators themselves and will be ignored further on. All computations were performed using the $\mathrm{R}$ software environment (R Development Core Team, 2009).

\section{1. fBm Generation}

The algorithm to generate one sample path of the length $n$, using the circulant matrix embedding method, is as follows:

- Choose $M=2^{p} \geqslant 2(n-1)$. Define the $M$-vector

$$
V=\left(r(0), r(1), \ldots, r\left(\frac{M}{2}-1\right), r\left(\frac{M}{2}\right), r\left(\frac{M}{2}-1\right), \ldots, r(2), r(1)\right),
$$

where

$$
r(k)=\frac{1}{2 n^{2 H}}\left[|k+1|^{2 H}-2 k^{2 H}+|k-1|^{2 H}\right]
$$

is the autocovariance function of the fractional Gaussian noise. 
- Compute $W=\left(w_{1}, \ldots, w_{M}\right)$, the fast Fourier transformation of $V$. All the coordinates of $W$ must be non-negative. If this is not the case, the value of $p$ must be increased until this requirement is met.

- Generate $U_{j}, V_{j} \sim \mathcal{N}(0,1)$ for all $1 \leqslant j<\frac{M}{2}$ and let $Z_{1}=U_{1}, Z_{\frac{M}{2}+1}=V_{1}$,

$$
Z_{j}=\frac{1}{\sqrt{2}}\left(U_{j}+i V_{j}\right), \quad Z_{M+2-j}=\frac{1}{\sqrt{2}}\left(U_{j}-i V_{j}\right), \quad 1<j \leqslant \frac{M}{2} .
$$

Then, define the $M$-vector $U$ as

$$
U_{k}=\sqrt{w_{k}} Z_{k}, \quad k=1, \ldots, M .
$$

- Compute $Y$ as an inverse fast Fourier transformation of the complex vector $U$ and define $X$ as

$$
X_{k}=X_{k-1}+\Re\left(Y_{k}\right), \quad X_{0}=0, k=1, \ldots, n,
$$

$\Re(Y)$ denoting the real part of the complex variable $Y$.

The obtained vector $X$ is the desired sample path of the fractional Brownian motion with the Hurst index $H$.

\subsection{Estimators}

1.2.1. Discrete Variation Estimators

For a real-valued process $X=\left\{X_{t}, t \in[0,1]\right\}$, we define the first and second order quadratic variations as

$$
V_{n}^{(1)}(X, 2)=\sum_{k=1}^{n}\left(\Delta_{k}^{(1)} X\right)^{2}, \quad V_{n}^{(2)}(X, 2)=\sum_{k=1}^{n-1}\left(\Delta_{k}^{(2)} X\right)^{2},
$$

where

$$
\begin{aligned}
& \Delta_{k}^{(1)} X=X\left(t_{k}^{n}\right)-X\left(t_{k-1}^{n}\right), \\
& \Delta_{k}^{(2)} X=X\left(t_{k+1}^{n}\right)-2 X\left(t_{k}^{n}\right)+X\left(t_{k-1}^{n}\right), \quad t_{k}^{n}=\frac{k}{n} .
\end{aligned}
$$

Let $X$ be the solution of (1). It is known (see, Kubilius and Melichov, 2008, 2009, 2010) that

$$
\widehat{H}_{\mathrm{dv} 1}^{n}=\frac{1}{2}-\frac{1}{2 \ln 2} \ln \frac{V_{2 n}^{(1)}(X, 2)}{V_{n}^{(1)}(X, 2)}, \quad \widehat{H}_{\mathrm{dv} 2}^{n}=\frac{1}{2}-\frac{1}{2 \ln 2} \ln \frac{V_{2 n}^{(2)}(X, 2)}{V_{n}^{(2)}(X, 2)}
$$

are strongly consistent estimators of the Hurst index $H$, i.e.,

$$
\widehat{H}_{\mathrm{dv} 1}^{n}-H \stackrel{\text { a.s. }}{\longrightarrow} 0 \quad \text { and } \quad \widehat{H}_{\mathrm{dv} 2}^{n}-H \stackrel{\text { a.s. }}{\longrightarrow} 0 \quad \text { as } n \rightarrow \infty .
$$


Here $V_{2 n}^{(\cdot)}(X, 2)$ corresponds to the quadratic variation of the whole sample path while $V_{n}^{(\cdot)}(X, 2)$ is the variation of the subset $\left\{X_{k}: k=2 j, 0 \leqslant j \leqslant[n / 2]\right\}$ where $[x]$ denotes the integer part of $x$.

\subsubsection{Gladyshev and $\eta$-Summing Oscillation Estimators}

The following estimators were described in Norvaiša and Salopek (2002). The ordinary least squares (OLS) Gladyshev and $\eta$-summing oscillation estimators require a sample path of the length $2^{n}+1, n \in \mathbb{N}$, which dictated the length of our modeled sample paths. Define $\eta_{M}=\left\{N_{m}=2^{m}: 1 \leqslant m \leqslant M\right\}$ and let

$$
s(m)=\sum_{i=1}^{N_{m}}\left[X\left(\frac{i}{N_{m}}\right)-X\left(\frac{i-1}{N_{m}}\right)\right]^{2} .
$$

The naive Gladyshev estimator of the Hurst index $\mathrm{H}$ is given by

$$
\widehat{H}_{\mathrm{gn}}^{M}=\frac{\log \sqrt{s(M) 2^{-M}}}{\log 2^{-M}},
$$

and the OLS Gladyshev estimator is given by

$$
\widehat{H}_{\mathrm{go}}^{M}=\frac{\sum_{m=1}^{M}\left(z_{m}-\bar{z}\right)^{2}}{\sum_{m=1}^{M}\left(z_{m}-\bar{z}\right) m},
$$

where $z_{m}=\log _{2} \sqrt{2^{m} / s(m)}$ for $m \in[1, \ldots, M]$ and $\bar{z}=M^{-1} \sum_{m=1}^{M} z_{m}$.

For every $m \in[1, \ldots, M]$, define

$$
Q(m)=\sum_{i=1}^{N_{m}}\left[\max _{t_{k}^{n} \in \Delta_{i, m}}\left\{X\left(t_{k}^{n}\right)\right\}-\min _{t_{k}^{n} \in \Delta_{i, m}}\left\{X\left(t_{k}^{n}\right)\right\}\right],
$$

where

$$
\Delta_{i, m}=\left[\frac{i-1}{N_{m}} ; \frac{i}{N_{m}}\right] .
$$

The naive oscillation estimator is defined by

$$
\widehat{H}_{\mathrm{osn}}^{M}=\frac{\log _{2}\left(N_{M} / Q(M)\right)}{\log _{2} N_{M}},
$$

and the OLS oscillation estimator is defined by

$$
\widehat{H}_{\mathrm{oso}}^{M}=\frac{\sum_{m=1}^{M}\left(z_{m}-\bar{z}\right)^{2}}{\sum_{m=1}^{M}\left(z_{m}-\bar{z}\right) N_{m}},
$$

where $z_{m}=\log _{2} \sqrt{N_{m} / Q(m)}$ and $\bar{z}=M^{-1} \sum_{m=1}^{M} z_{m}$.

For $M=14$ we simulate estimates defined above. 


\subsubsection{Variogram Estimator}

The variogram of the process $X=\left\{X_{t}, t \in[0,1]\right\}$ for the lag $l$ is defined (Chronopoulou and Viens, 2010) as

$$
V(l)=\mathbb{E}\left[\left(X_{t}-X_{t-l}\right)^{2}\right]
$$

In order to estimate the Hurst index $H$, we choose a set of lags, in our case, it was $\left\{l=2^{i}, i=0, \ldots, 5\right\}$. Then $\widehat{H}_{\mathrm{var}}^{n}=b / 2$, where $b$ is the slope of the linear regression line of $\log (V(l))$ against $\log (l)$.

\subsubsection{IR Estimator}

This estimator was proposed by Bardet and Surgailis (2010). For the O-U or gBm process $X=\left\{X_{t}, t \in[0,1]\right\}$ given at points $t_{k}^{n}=k / n, k=0,1, \ldots, n$, the IR estimator of $H$ can be computed using the approximated formula below

$$
\widehat{H}_{\mathrm{ir}}^{n}=\frac{1}{0.1468}\left(\frac{1}{n-2} \sum_{k=1}^{n-2} \frac{\left|\Delta_{k}^{(2)} X+\Delta_{k+1}^{(2)} X\right|}{\left|\Delta_{k}^{(2)} X\right|+\left|\Delta_{k+1}^{(2)} X\right|}-0.5174\right),
$$

where $\Delta_{k}^{(2)} X=X\left(t_{k+1}^{n}\right)-2 X\left(t_{k}^{n}\right)+X\left(t_{k-1}^{n}\right)$.

\section{Estimation for the Ornstein-Uhlenbeck Process}

\subsection{Dependance on the Value of the Hurst Index}

The first goal of this paper is to compare the behavior of these estimators for different values of the Hurst index $H$. Table 1 presents the biases $\bar{H}-H=\mathbf{E}(\widehat{H}-H)$ as well as the mean squared errors defined as $\operatorname{MSE}(\widehat{H})=\mathbf{E}(\widehat{H}-H)^{2}$ for the sample path lengths of, respectively, $2^{14}+1$ and $2^{10}+1$ points. Figure 1 illustrates this further presenting the boxplots of the considered estimators for the length of sample paths $n=2^{14}+1$ points. Here and further in this paper the figures related to the estimators $\widehat{H}_{\mathrm{gn}}$ and $\widehat{H}_{\text {go }}$ are omitted, since their behavior does not significantly differ from the behavior of $\widehat{H}_{\text {osn }}$ and $\widehat{H}_{\text {oso }}$. The numbers printed in bold correspond to the estimators that performed better than the others for the specific value of $H$ and the measure considered.

It can be seen that the estimators $\widehat{H}_{\mathrm{dv} 1}, \widehat{H}_{\mathrm{var}}, \widehat{H}_{\mathrm{gn}}$ and $\widehat{H}_{\mathrm{osn}}$ exhibit increases of the biases and the mean squared errors for larger values of $H$. $\widehat{H}_{\text {go }}$ and $\widehat{H}_{\text {oso }}$ seem to be less dependant on that, however, they tend to slightly undervalue the Hurst index when it is close to $1 . \widehat{H}_{\text {ir }}$ tends to slightly undervalue $H$ when $H<3 / 4$ and to overvalue it when $H>3 / 4$; the most likely cause of this are the numerical constants in the formula used for this estimator. The behavior of $\widehat{H}_{\mathrm{dv} 2}$ does not change noticeably for different values of $H$.

Another interesting observation is the, comparatively, very low mean squared errors of $\widehat{H}_{\mathrm{gn}}$ and $\widehat{H}_{\mathrm{osn}}$ which they display as long as the Hurst index is not too close to 1 . 
Table 1

Comparison of the estimators for the O-U process

\begin{tabular}{llrrrr}
\hline$H$ & & 0.55 & 0.7 & 0.8 & 0.95 \\
\hline MSE & dv1 & 0.008 & $\mathbf{0 . 0 0 5}$ & $\mathbf{0 . 0 0 8}$ & 0.020 \\
& dv2 & 0.015 & 0.011 & 0.011 & $\mathbf{0 . 0 1 0}$ \\
& var & $\mathbf{0 . 0 0 7}$ & 0.009 & 0.014 & 0.024 \\
gn & $\mathbf{0 . 0 0 2}$ & $\mathbf{0 . 0 0 2}$ & $\mathbf{0 . 0 0 3}$ & $\mathbf{0 . 0 2 1}$ \\
& osn & $\mathbf{0 . 0 0 4}$ & $\mathbf{0 . 0 0 4}$ & $\mathbf{0 . 0 0 5}$ & 0.025 \\
go & 0.038 & 0.037 & 0.048 & 0.050 \\
& oso & 0.041 & 0.037 & 0.050 & 0.053 \\
& ir & 0.022 & 0.019 & 0.019 & 0.022 \\
\hline$H-H$ & dv1 & $\mathbf{0 . 0 0 0}$ & $\mathbf{0 . 0 0 1}$ & $-\mathbf{0 . 0 0 1}$ & $-\mathbf{0 . 0 0 9}$ \\
& dv2 & $\mathbf{0 . 0 0 0}$ & $\mathbf{0 . 0 0 1}$ & -0.002 & $-\mathbf{0 . 0 0 1}$ \\
& var & $\mathbf{0 . 0 0 0}$ & $-\mathbf{0 . 0 0 1}$ & $\mathbf{0 . 0 0 0}$ & $-\mathbf{0 . 0 1 2}$ \\
gn & 0.037 & 0.037 & 0.037 & 0.041 \\
osn & 0.060 & 0.060 & 0.060 & 0.063 \\
go & -0.013 & -0.024 & -0.019 & -0.032 \\
oso & -0.004 & 0.017 & -0.009 & -0.020 \\
ir & -0.019 & -0.009 & -0.001 & 0.028 \\
\hline
\end{tabular}

(a) $N=2^{14}+1$

\begin{tabular}{lrrrr}
\hline$H$ & 0.55 & 0.7 & 0.8 & 0.95 \\
\hline dv1 & 0.027 & 0.024 & $\mathbf{0 . 0 2 6}$ & $\mathbf{0 . 0 3 0}$ \\
dv2 & 0.050 & 0.054 & 0.050 & 0.044 \\
var & 0.029 & 0.031 & 0.038 & $\mathbf{0 . 0 4 0}$ \\
gn & $\mathbf{0 . 0 0 6}$ & $\mathbf{0 . 0 0 6}$ & $\mathbf{0 . 0 1 0}$ & $\mathbf{0 . 0 4 0}$ \\
osn & $\mathbf{0 . 0 1 0}$ & $\mathbf{0 . 0 1 1}$ & $\mathbf{0 . 0 1 5}$ & 0.048 \\
go & 0.092 & 0.061 & 0.071 & 0.068 \\
oso & 0.093 & 0.060 & 0.073 & 0.071 \\
ir & 0.074 & 0.067 & 0.069 & 0.077 \\
\hline dv1 & $\mathbf{0 . 0 0 0}$ & $-\mathbf{0 . 0 0 4}$ & $-\mathbf{0 . 0 0 1}$ & $-\mathbf{0 . 0 1 6}$ \\
dv2 & $\mathbf{0 . 0 0 1}$ & $\mathbf{0 . 0 0 0}$ & $-\mathbf{0 . 0 0 2}$ & $\mathbf{0 . 0 0 8}$ \\
var & $\mathbf{0 . 0 0 0}$ & -0.012 & -0.007 & -0.025 \\
gn & 0.051 & 0.052 & 0.052 & 0.062 \\
osn & 0.084 & 0.084 & 0.084 & 0.091 \\
go & -0.010 & -0.035 & -0.030 & -0.044 \\
oso & $\mathbf{0 . 0 0 3}$ & $-\mathbf{0 . 0 2 3}$ & -0.014 & $-\mathbf{0 . 0 2 8}$ \\
ir & -0.009 & $-\mathbf{0 . 0 0 4}$ & $-\mathbf{0 . 0 0 4}$ & 0.039 \\
\hline
\end{tabular}

(b) $N=2^{10}+1$

However these estimators also possess the largest bias. $\widehat{H}_{\text {go }}$ and $\widehat{H}_{\text {oso }}$, the OLS versions of these two estimators behave in a completely different way - they have smaller biases which are comparable to those of the other considered estimators, but this comes at the cost of a heavily increased MSE.

\subsection{Dependance on the Length of the Sample Path}

The second goal of this paper is to compare the behavior of these estimators for different lengths of sample paths as well as to illustrate how the estimators' variances fluctuate as the length of sample paths is increased. Table 2 shows the mean squared errors and the biases for the Hurst index values of 0.65 and 0.85 , respectively. Figure 2 boxplots of the estimators for $H=0.85$.

The first obvious observation is that the bias of $\widehat{H}_{\mathrm{gn}}$ and $\widehat{H}_{\text {osn }}$ increases as the length of sample paths is decreased. $\widehat{H}_{\text {go }}$ and $\widehat{H}_{\text {oso }}$ do not share this property, however their mean squared errors display only minor decreases when longer sample path lengths are taken. The other estimators show a rather regular decrease of their mean squared errors which is further illustrated by Fig. 3 presenting the plots of $\log (S D)$ against $\log (n)$ for $H \in\{0.55,0.6, \ldots, 0.95\}$ where SD denotes the standard deviations.

Figure 3 shows the rate at which the standard deviation decreases as the sample path length is increased. It can be seen that this rate depends on the value of $H$ for all the estimators except $\widehat{H}_{\mathrm{dv} 2}$ and $\widehat{H}_{\mathrm{ir}}$. The general trend is that this rate is lower for higher 

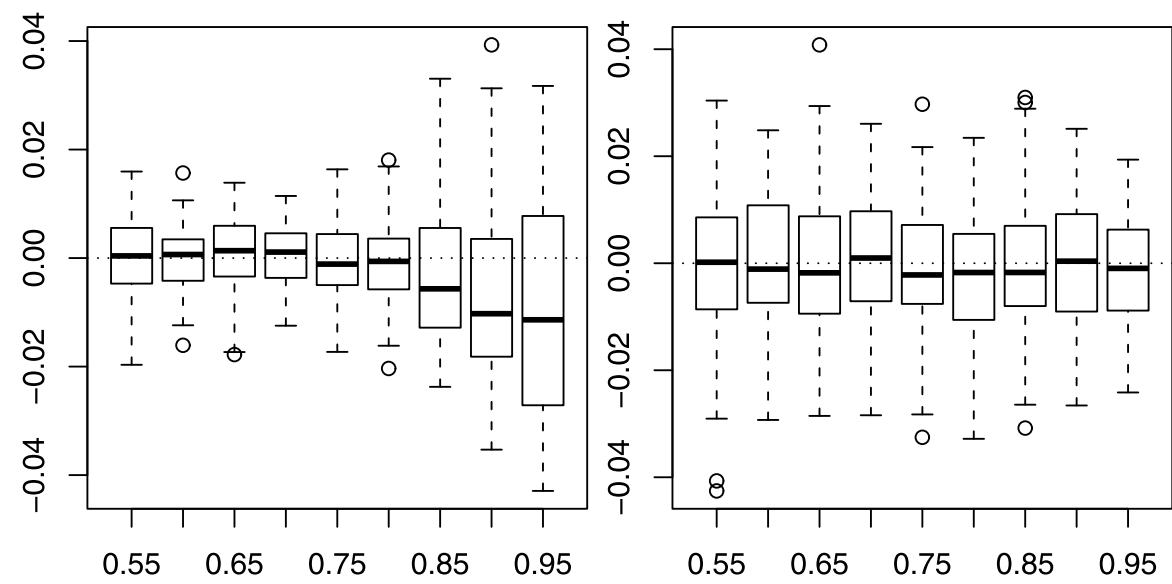

(a) dv1

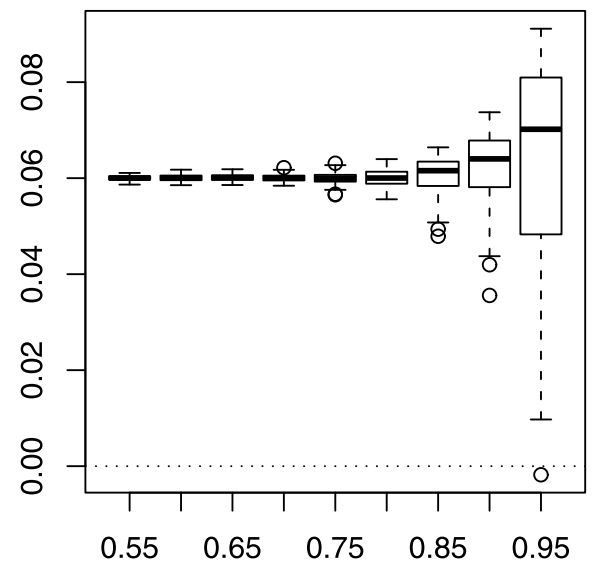

(b) dv2

(c) gn

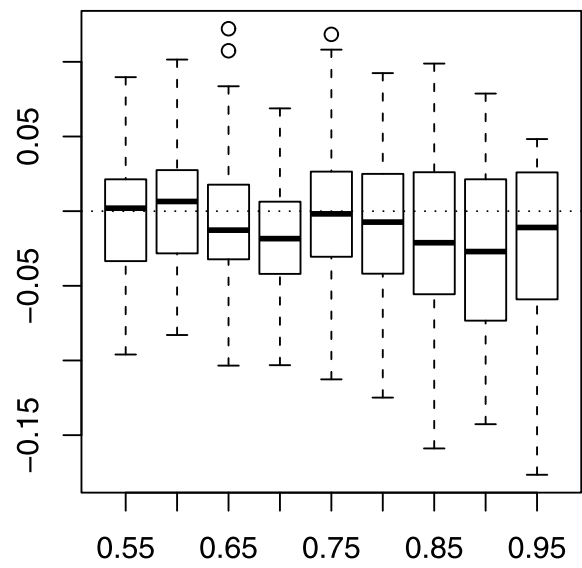

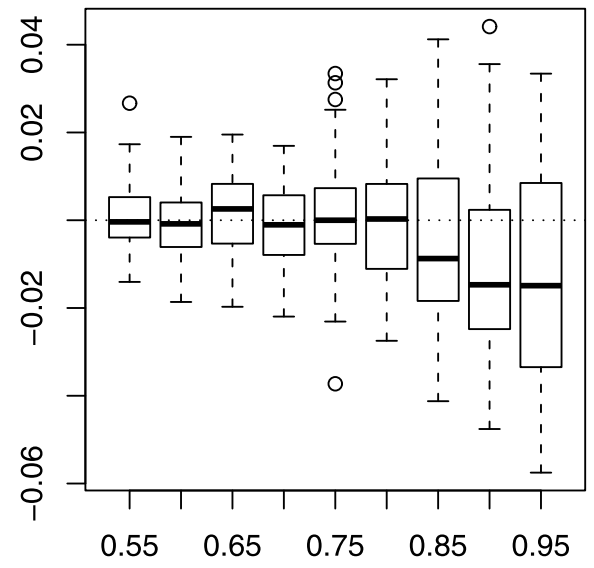

(e) var (d) osn

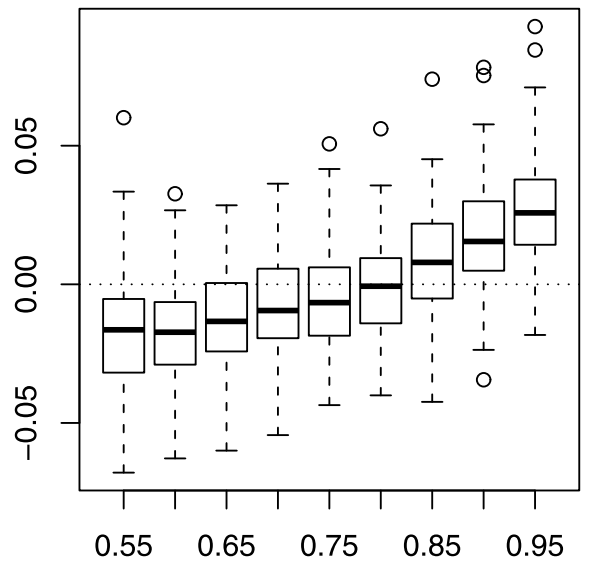

(f) ir

Fig. 1. Boxplots of the estimators for the O-U process of sample path length $n=2^{14}+1$. 

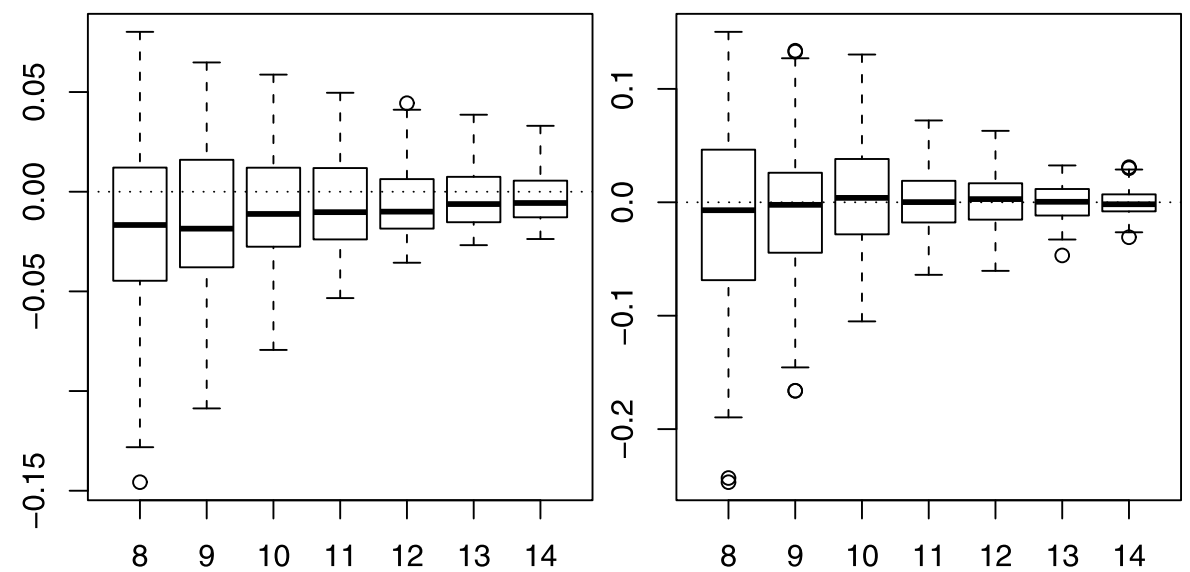

(a) dv1

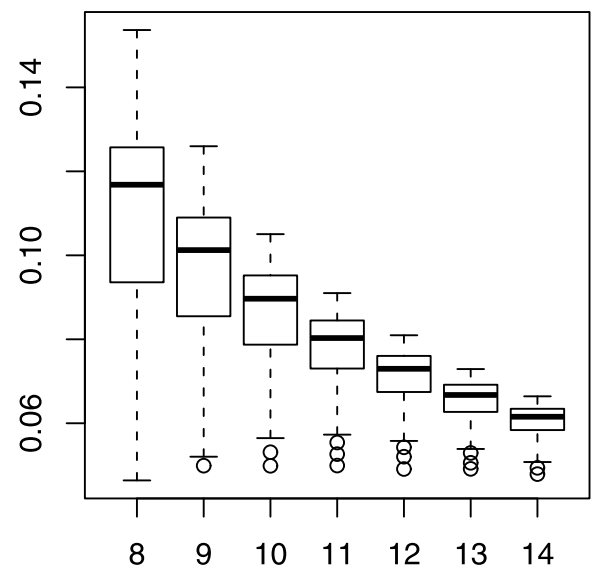

(b) dv2

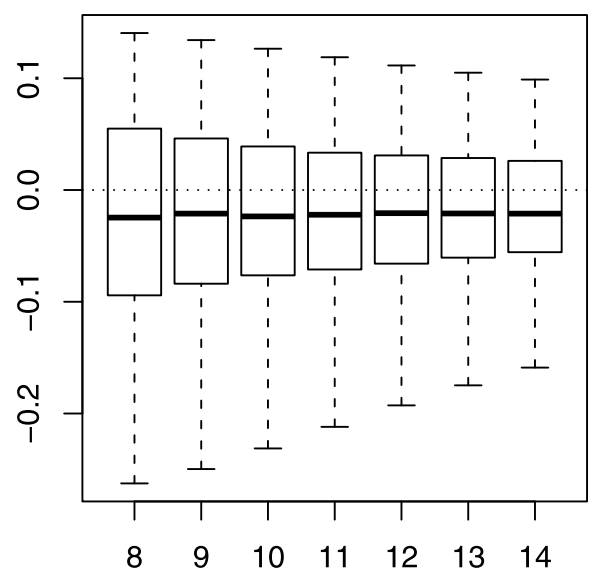

(c) osn

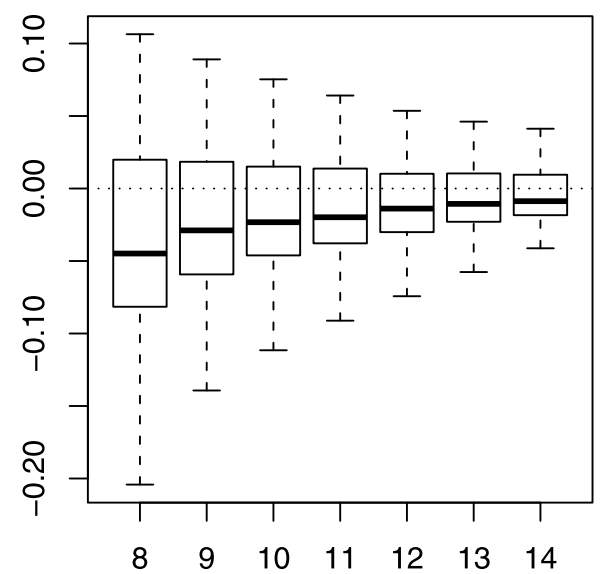

(d) oso

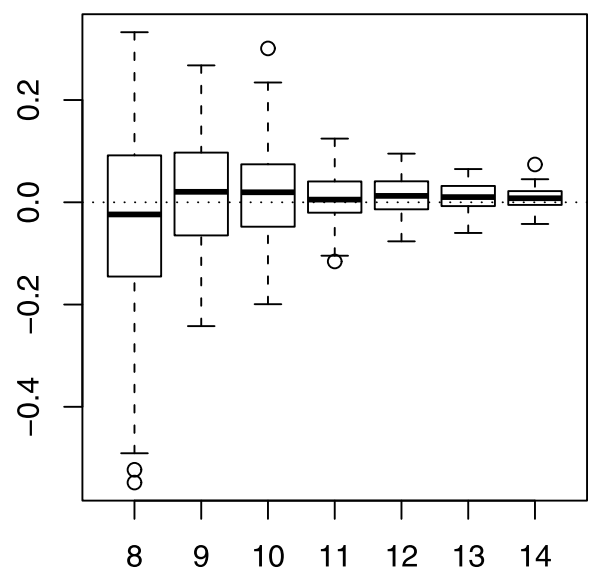

(e) var

(f) ir

Fig. 2. Boxplots of the estimators for the O-U process for $H=0.85, n=2^{k}, k=8, \ldots, 14$. 


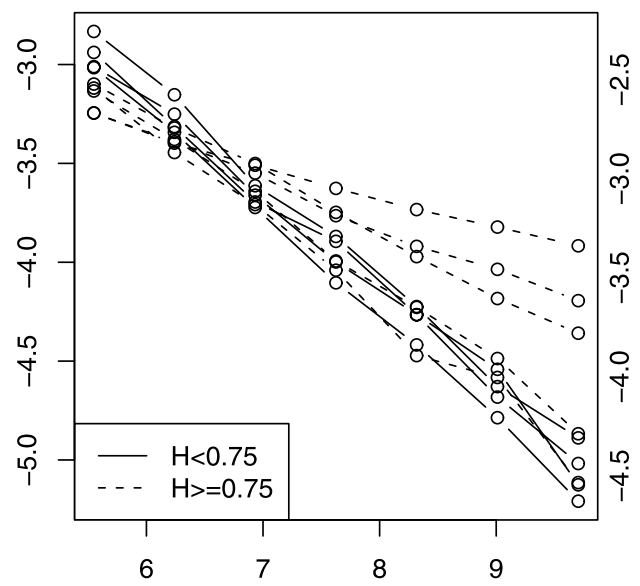

(a) dv1

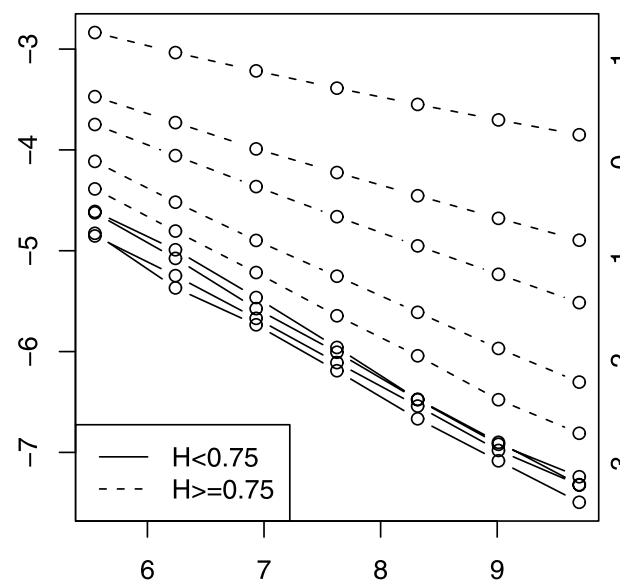

(c) osn

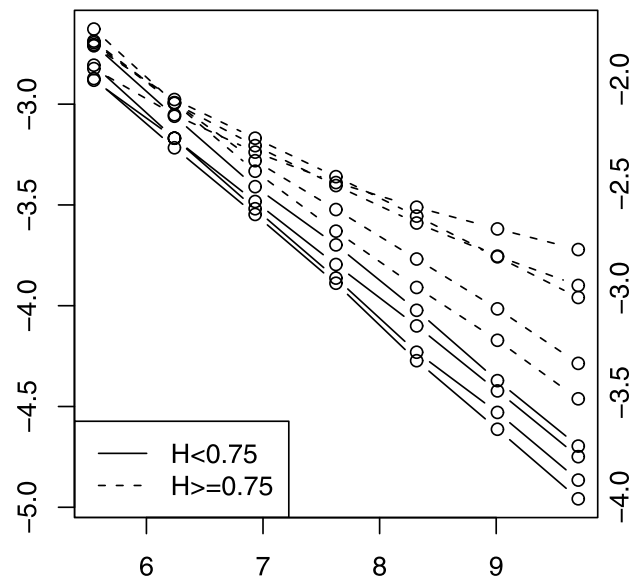

(e) var

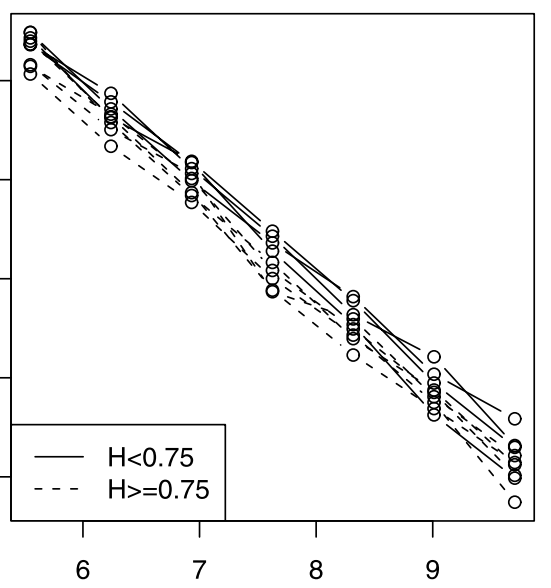

(b) dv2

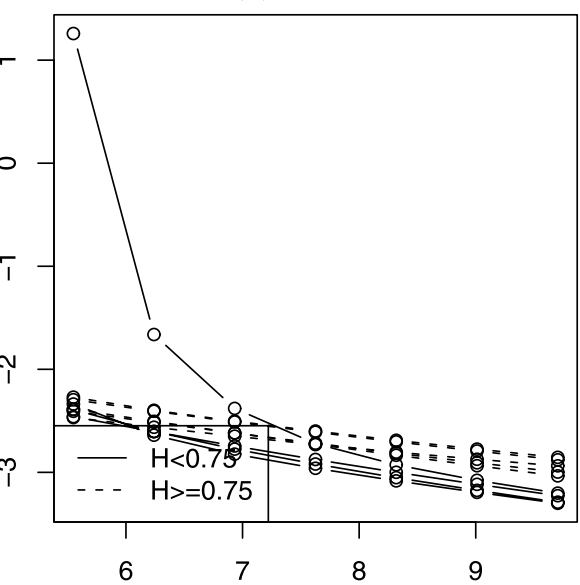

(d) oso

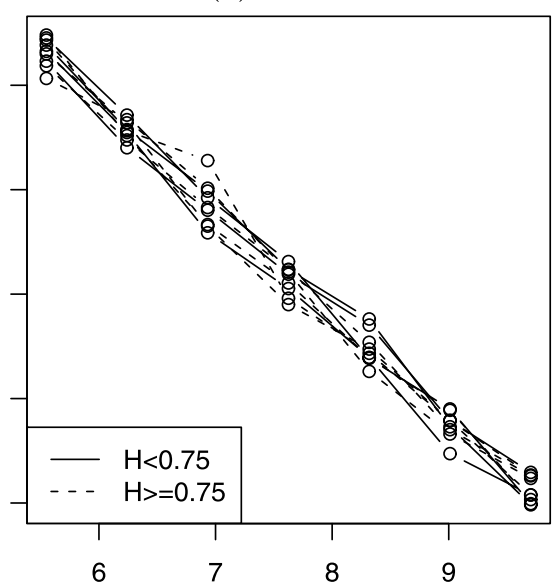

(f) ir

Fig. 3. Dependance of $\log (S D)$ against $\log (n)$ for the O-U process, $H \in\{0.55,0.6, \ldots, 0.95\}$. 
Table 2

Comparison of the estimators for the O-U process for sample path lengths $N=2^{k}+1$

\begin{tabular}{llllll}
\hline & $k$ & \multicolumn{1}{l}{10} & \multicolumn{1}{l}{12} & \multicolumn{1}{l}{14} \\
\hline MSE & dv1 & $\mathbf{0 . 0 5 3}$ & $\mathbf{0 . 0 2 5}$ & $\mathbf{0 . 0 1 4}$ & $\mathbf{0 . 0 0 7}$ \\
& dv2 & 0.102 & 0.055 & 0.027 & 0.013 \\
& var & 0.067 & 0.033 & 0.018 & 0.009 \\
gn & $\mathbf{0 . 0 1 3}$ & $\mathbf{0 . 0 0 6}$ & $\mathbf{0 . 0 0 3}$ & $\mathbf{0 . 0 0 2}$ \\
& osn & $\mathbf{0 . 0 2 1}$ & $\mathbf{0 . 0 1 1}$ & $\mathbf{0 . 0 0 6}$ & $\mathbf{0 . 0 0 4}$ \\
go & 0.084 & 0.063 & 0.049 & 0.039 \\
oso & 0.086 & 0.064 & 0.050 & 0.040 \\
ir & 0.172 & 0.079 & 0.037 & 0.018 \\
\hline $\bar{H}-H$ & dv1 & $-\mathbf{0 . 0 0 5}$ & $\mathbf{0 . 0 0 0}$ & $\mathbf{0 . 0 0 2}$ & $\mathbf{0 . 0 0 1}$ \\
& dv2 & $-\mathbf{0 . 0 1 6}$ & $\mathbf{0 . 0 0 0}$ & $\mathbf{0 . 0 0 1}$ & $\mathbf{0 . 0 0 0}$ \\
var & -0.021 & $-\mathbf{0 . 0 0 3}$ & $-\mathbf{0 . 0 0 1}$ & $\mathbf{0 . 0 0 1}$ \\
gn & 0.064 & 0.051 & 0.043 & 0.037 \\
osn & 0.105 & 0.084 & 0.070 & 0.060 \\
go & -0.036 & -0.028 & -0.021 & -0.016 \\
oso & $-\mathbf{0 . 0 1 7}$ & -0.014 & -0.011 & -0.009 \\
ir & -0.043 & -0.013 & -0.014 & -0.013 \\
\hline
\end{tabular}

(a) $H=0.65$

\begin{tabular}{lrrrr}
\hline$k$ & \multicolumn{1}{l}{8} & \multicolumn{1}{l}{10} & \multicolumn{1}{l}{12} & \multicolumn{1}{l}{14} \\
\hline dv1 & $\mathbf{0 . 0 4 5}$ & $\mathbf{0 . 0 3 0}$ & $\mathbf{0 . 0 1 9}$ & 0.013 \\
dv2 & 0.089 & 0.051 & 0.025 & $\mathbf{0 . 0 1 2}$ \\
var & 0.068 & 0.042 & 0.029 & 0.019 \\
gn & $\mathbf{0 . 0 2 8}$ & $\mathbf{0 . 0 1 5}$ & $\mathbf{0 . 0 0 9}$ & $\mathbf{0 . 0 0 5}$ \\
osn & $\mathbf{0 . 0 3 6}$ & $\mathbf{0 . 0 2 0}$ & $\mathbf{0 . 0 1 2}$ & $\mathbf{0 . 0 0 8}$ \\
go & 0.095 & 0.077 & 0.063 & 0.053 \\
oso & 0.102 & 0.082 & 0.067 & 0.057 \\
ir & 0.170 & 0.095 & 0.038 & 0.021 \\
\hline dv1 & $-\mathbf{0 . 0 1 6}$ & $-\mathbf{0 . 0 0 8}$ & $-\mathbf{0 . 0 0 5}$ & $-\mathbf{0 . 0 0 3}$ \\
dv2 & $-\mathbf{0 . 0 1 5}$ & $\mathbf{0 . 0 0 5}$ & $-\mathbf{0 . 0 0 2}$ & $-\mathbf{0 . 0 0 1}$ \\
var & -0.038 & -0.018 & $-\mathbf{0 . 0 1 0}$ & $-\mathbf{0 . 0 0 5}$ \\
gn & 0.070 & 0.054 & 0.044 & 0.037 \\
osn & 0.110 & 0.086 & 0.071 & 0.061 \\
go & -0.054 & -0.043 & -0.035 & -0.028 \\
oso & -0.033 & -0.027 & -0.022 & -0.018 \\
ir & -0.039 & 0.019 & 0.011 & 0.008 \\
\hline
\end{tabular}

(b) $H=0.85$

values of $H$ which is most notable for $\widehat{H}_{\mathrm{gn}}$ and $\widehat{H}_{\mathrm{osn}}$. On the other hand $\widehat{H}_{\mathrm{dv} 2}$ and $\widehat{H}_{\mathrm{ir}}$ display no dependance of this kind. Also, if we consider the linear regression $\log (S D) \sim$ $\log (n)$ for these two estimators, its slope is -0.5003 for $\widehat{H}_{\mathrm{dv} 2}$ and -0.5013 for $\widehat{H}_{\mathrm{ir}}$, which suggests that for both these estimators $S D\left(\widehat{H}_{(\cdot)}\right) \sim \mathcal{O}\left(n^{-1 / 2}\right)$.

\section{Estimation of the geometric Brownian motion}

\subsection{Dependance on the Value of the Hurst Index}

Table 3 presents the mean squared errors and the biases for the sample path lengths of $2^{14}$ and $2^{10}$. Boxplots of these estimators for the sample path length $n=2^{14}$ can be found in Fig. 4. It can be seen that, for the non-Gaussian gBm process, the estimators $\widehat{H}_{\mathrm{gn}}$ and $\widehat{H}_{\text {osn }}$ display higher biases for all the values of $H$.

An interesting observation is that, in the case of the O-U process the mean squared errors of $\widehat{H}_{\mathrm{gn}}$ and $\widehat{H}_{\mathrm{osn}}$ were the lowest of all the considered estimators, while for the gBm model their mean squared errors surpassed those of $\widehat{H}_{\mathrm{dv} 1}, \widehat{H}_{\mathrm{dv} 2}$ and $\widehat{H}_{\mathrm{var}}$.

The behavior of $\widehat{H}_{\mathrm{dv} 2}$ and $\widehat{H}_{\text {ir }}$ does not display notable differences for these two processes. 
Table 3

Comparison of the estimators for the $\mathrm{gBm}$ process

\begin{tabular}{llrrrr}
\hline$H$ & & 0.55 & 0.7 & 0.8 & 0.95 \\
\hline MSE & dv1 & $\mathbf{0 . 0 0 8}$ & $\mathbf{0 . 0 0 6}$ & $\mathbf{0 . 0 0 9}$ & $\mathbf{0 . 0 2 1}$ \\
& dv2 & $\mathbf{0 . 0 1 6}$ & $\mathbf{0 . 0 1 2}$ & $\mathbf{0 . 0 1 1}$ & $\mathbf{0 . 0 1 0}$ \\
& var & $\mathbf{0 . 0 0 8}$ & $\mathbf{0 . 0 1 0}$ & 0.015 & 0.025 \\
gn & 0.032 & 0.030 & 0.034 & 0.049 \\
& osn & 0.035 & 0.032 & 0.037 & 0.054 \\
go & 0.033 & 0.046 & 0.053 & 0.049 \\
& oso & 0.035 & 0.049 & 0.056 & 0.053 \\
& ir & 0.022 & 0.019 & 0.019 & $\mathbf{0 . 0 2 2}$ \\
\hline$H-H$ & dv1 & $\mathbf{0 . 0 0 0}$ & $\mathbf{0 . 0 0 1}$ & $\mathbf{0 . 0 0 0}$ & $-\mathbf{0 . 0 0 6}$ \\
& dv2 & $\mathbf{0 . 0 0 0}$ & $\mathbf{0 . 0 0 1}$ & $-\mathbf{0 . 0 0 2}$ & $\mathbf{0 . 0 0 0}$ \\
& var & $\mathbf{0 . 0 0 0}$ & $\mathbf{0 . 0 0 1}$ & $\mathbf{0 . 0 0 0}$ & $-\mathbf{0 . 0 0 9}$ \\
gn & 0.055 & 0.051 & 0.061 & 0.059 \\
osn & 0.080 & 0.076 & 0.086 & 0.082 \\
go & -0.014 & -0.016 & -0.021 & -0.026 \\
oso & $-\mathbf{0 . 0 0 3}$ & -0.006 & -0.011 & -0.014 \\
ir & -0.019 & -0.009 & $-\mathbf{0 . 0 0 1}$ & 0.028 \\
\hline
\end{tabular}

(a) $N=2^{14}+1$

\begin{tabular}{lrrrr}
\hline$H$ & 0.55 & 0.7 & 0.8 & 0.95 \\
\hline dv1 & $\mathbf{0 . 0 3 0}$ & $\mathbf{0 . 0 2 6}$ & $\mathbf{0 . 0 2 9}$ & $\mathbf{0 . 0 3 0}$ \\
dv2 & 0.054 & 0.054 & 0.055 & $\mathbf{0 . 0 4 6}$ \\
var & $\mathbf{0 . 0 3 2}$ & 0.039 & 0.044 & 0.038 \\
gn & 0.047 & 0.044 & 0.051 & 0.079 \\
osn & 0.053 & 0.048 & 0.058 & 0.088 \\
go & 0.055 & 0.072 & 0.080 & 0.066 \\
oso & 0.057 & 0.077 & 0.084 & 0.070 \\
ir & 0.074 & 0.067 & 0.069 & 0.077 \\
\hline dv1 & $-\mathbf{0 . 0 0 1}$ & $-\mathbf{0 . 0 0 1}$ & $\mathbf{0 . 0 0 0}$ & $-\mathbf{0 . 0 1 2}$ \\
dv2 & $\mathbf{0 . 0 0 1}$ & $\mathbf{0 . 0 0 0}$ & $-\mathbf{0 . 0 0 2}$ & $\mathbf{0 . 0 0 7}$ \\
var & $\mathbf{0 . 0 0 0}$ & -0.006 & -0.008 & -0.020 \\
gn & 0.077 & 0.072 & 0.086 & 0.086 \\
osn & 0.112 & 0.106 & 0.120 & 0.116 \\
go & -0.020 & -0.027 & -0.036 & -0.038 \\
oso & $-\mathbf{0 . 0 0 1}$ & -0.009 & -0.019 & -0.020 \\
ir & -0.009 & -0.002 & $-\mathbf{0 . 0 0 4}$ & 0.039 \\
\hline
\end{tabular}

(b) $N=2^{10}+1$

\subsection{Dependance on the Length of the Sample Path}

Table 4 presents the mean squared errors and the biases for $H=0.65$ and $H=0.85$. Figure 5 shows the boxplots of the estimators considered for $H=0.85$, while Fig. 6 presents the plots of $\log (S D)$ against $\log (n)$.

Compared to the O-U case the biases of $\widehat{H}_{\text {gn }}$ and $\widehat{H}_{\text {osn }}$ are higher for all sample path lengths. The mean squared errors of $\widehat{H}_{\mathrm{gn}}, \widehat{H}_{\mathrm{osn}}, \widehat{H}_{\mathrm{go}}$ and $\widehat{H}_{\text {oso }}$ are higher for all sample path lengths. In the case of relatively short sample paths $\left(2^{8}-2^{10}\right)$ and $H>3 / 4$, the estimators $\widehat{H}_{\text {go }}$ and $\widehat{H}_{\text {oso }}$ have at times severely overestimated the Hurst index $H$ with the estimated value being higher than 2 . Those values were excluded from their boxplots. The slope of the linear regression $\log (S D) \sim \log (n)$ is -0.5015 for $\widehat{H}_{\mathrm{dv} 2}$ and -0.5011 for $\widehat{H}_{\mathrm{ir}}$, which does not differ significantly from the O-U case.

\section{Conclusions}

- The estimators $\widehat{H}_{\text {gn }}$ and $\widehat{H}_{\text {osn }}$, despite showing the least mean squared errors in the $\mathrm{O}-\mathrm{U}$ case, have also shown much higher biases than other estimators considered in this paper. This bias increases as the sample path length is decreased but shows no dependance on the value of the Hurst index $H$ as long as $H$ is not too close to 1. When $H>0.9$, this bias increases further. In the $\mathrm{gBm}$ case the mean squared errors of these two estimators were greater than those of $\widehat{H}_{\mathrm{dv} 1}, \widehat{H}_{\mathrm{dv} 2}$ and $\widehat{H}_{\mathrm{var}}$. 

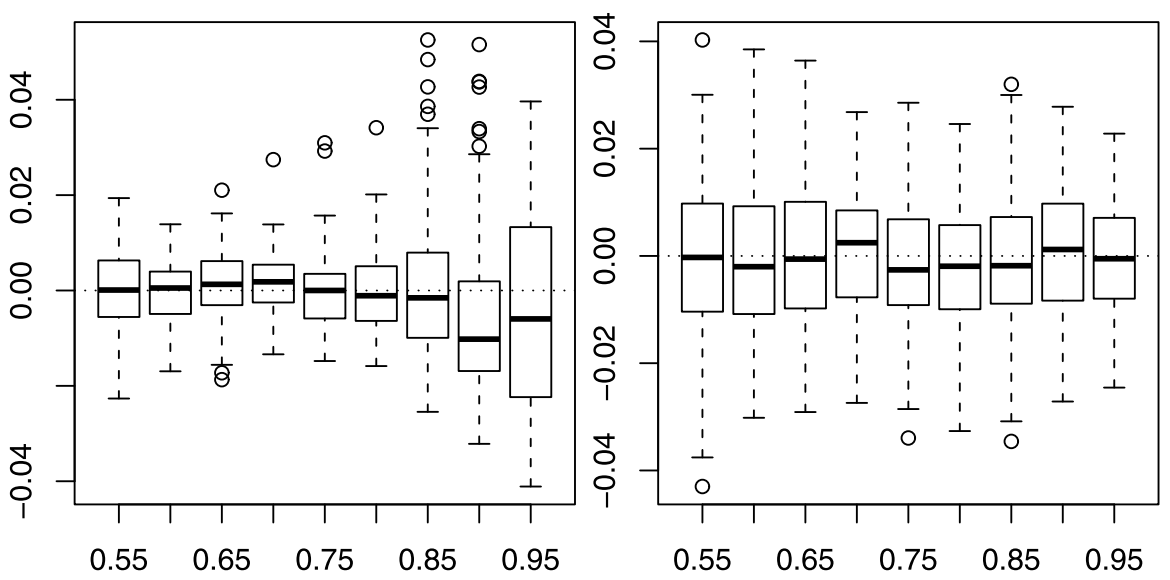

(a) dv1

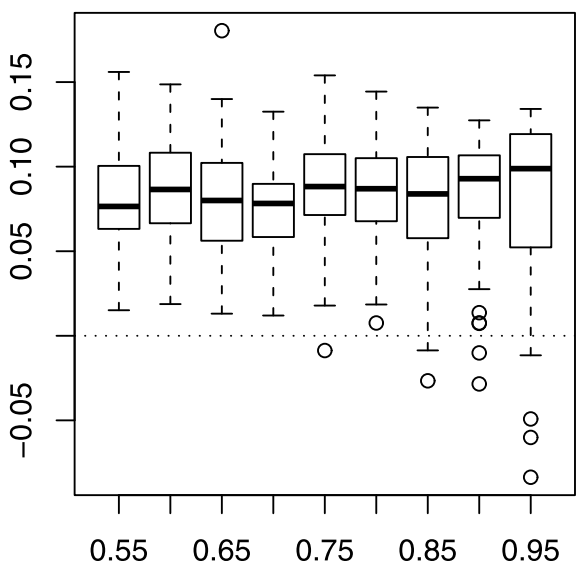

(b) dv2

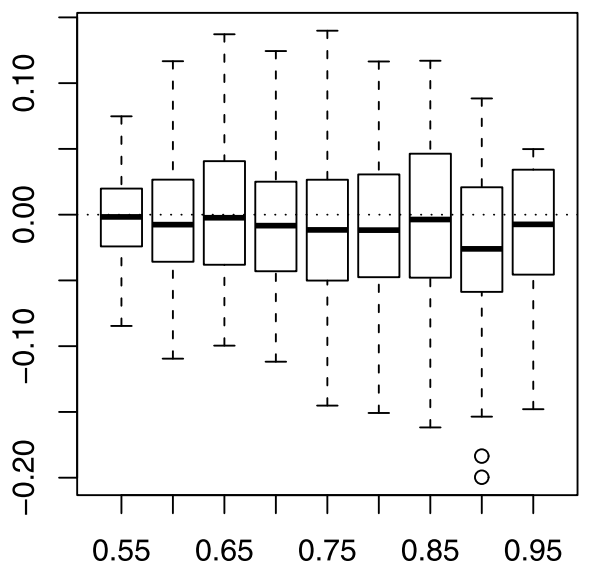

(c) osn

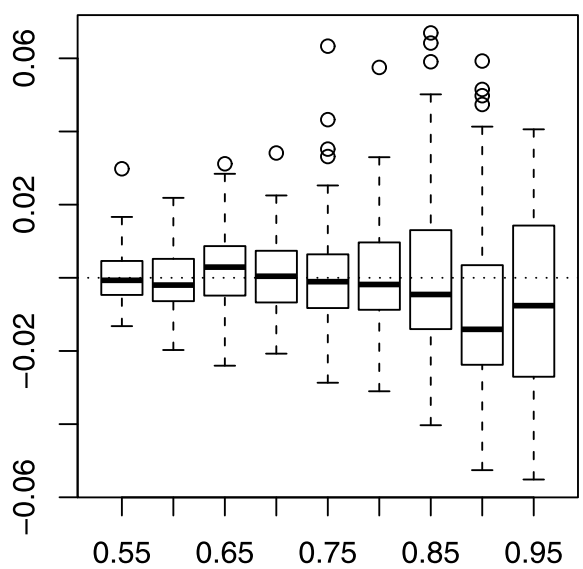

(d) oso

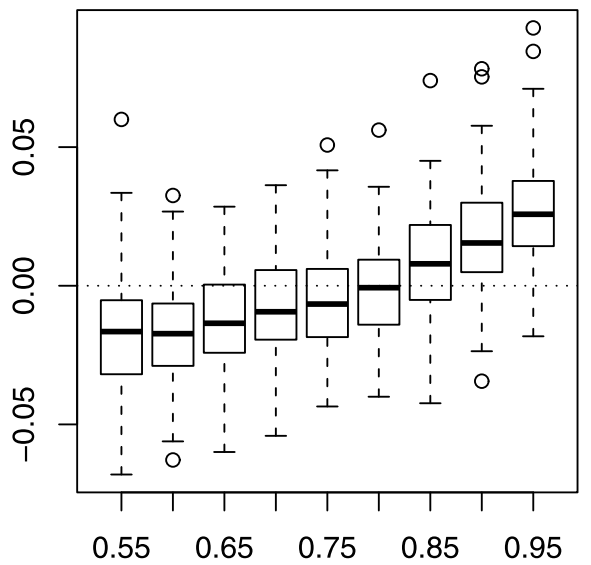

(e) $\operatorname{var}$

(f) ir

Fig. 4. Boxplots of the estimators for the $\mathrm{gBm}$ process for sample path length $n=2^{14}+1$. 


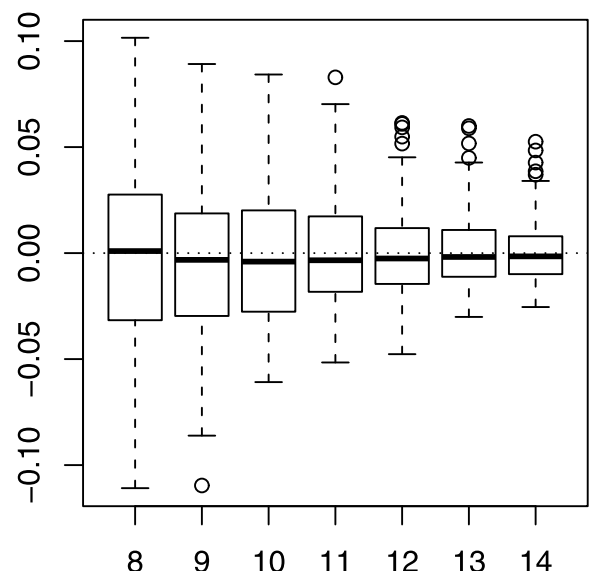

(a) dv1

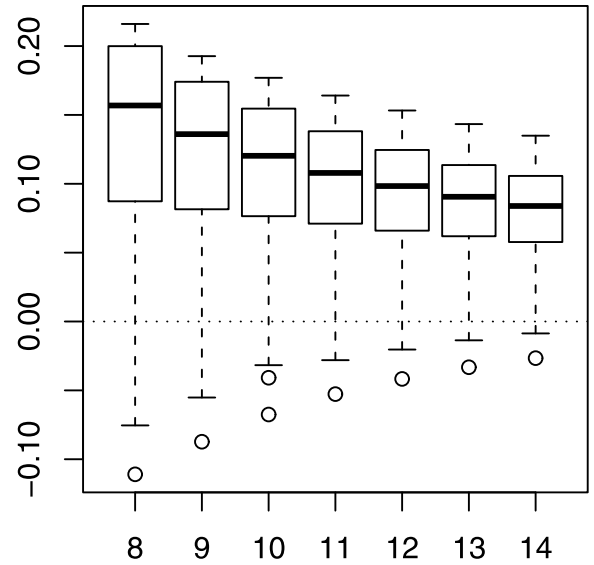

(c) osn

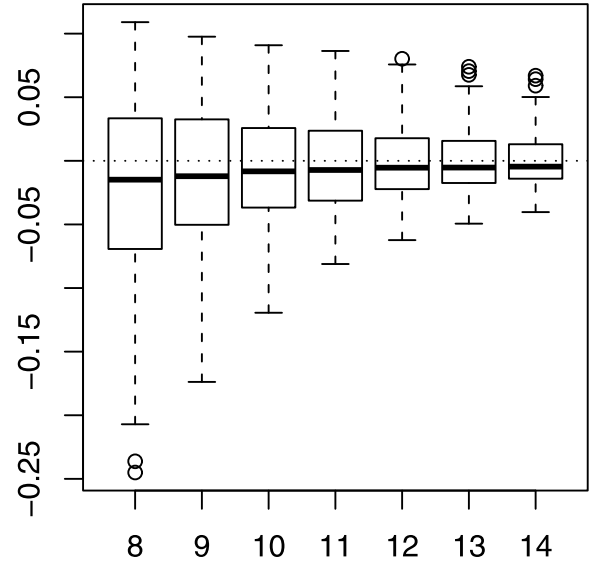

(e) var

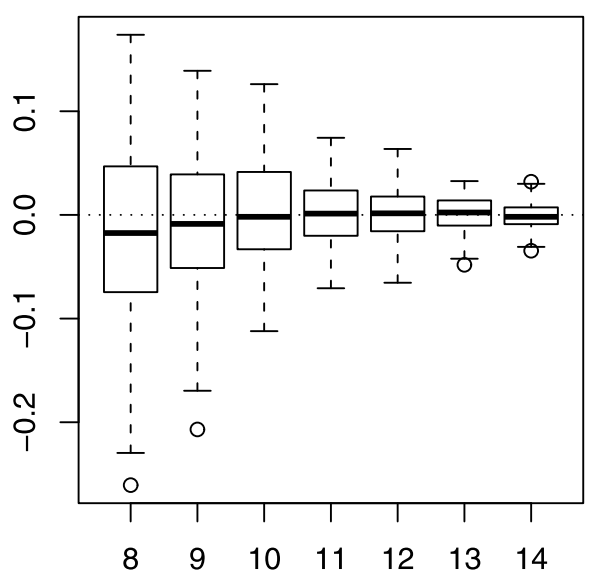

(b) dv2

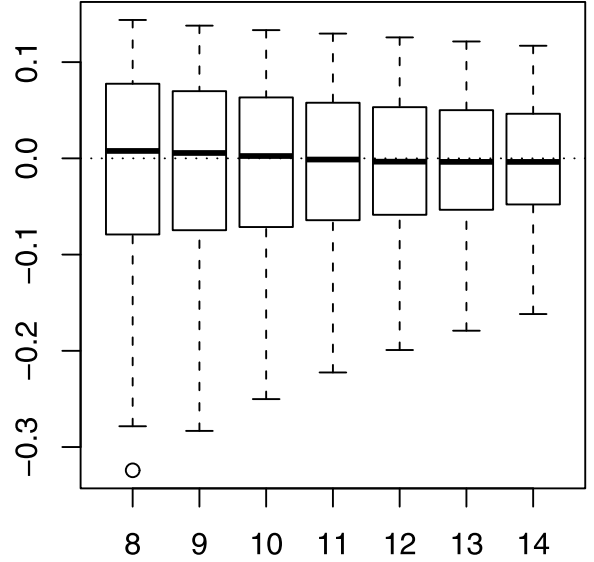

(d) oso

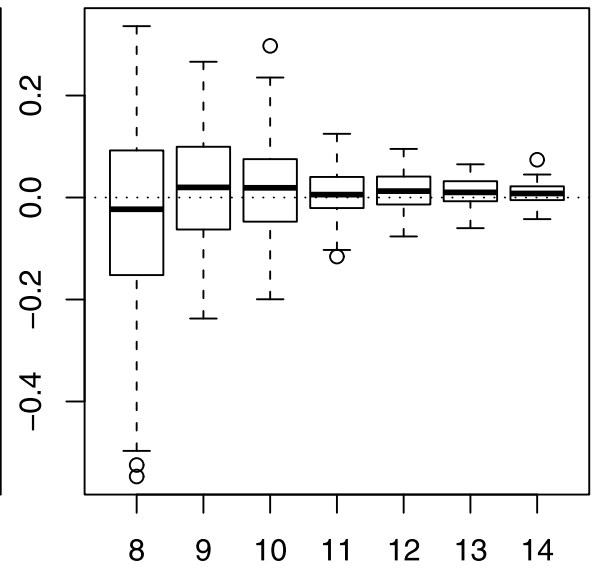

(f) ir

Fig. 5. Boxplots of the estimators for the $\mathrm{gBm}$ process for $H=0.85, n=2^{k}, k=8, \ldots, 14$. 

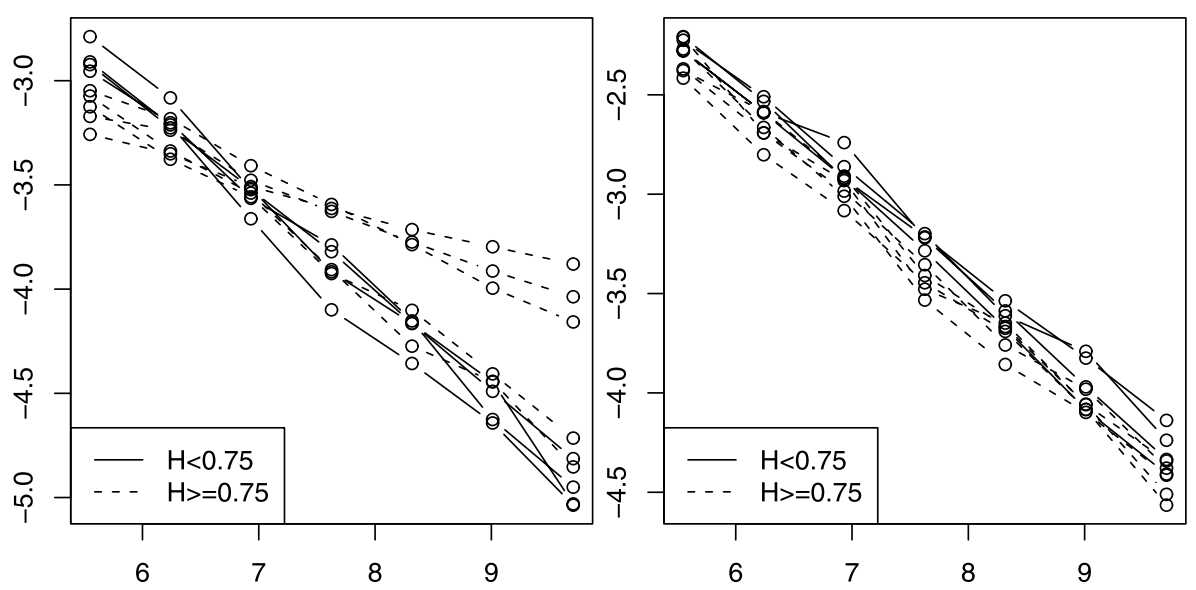

(a) dv1

(b) dv2
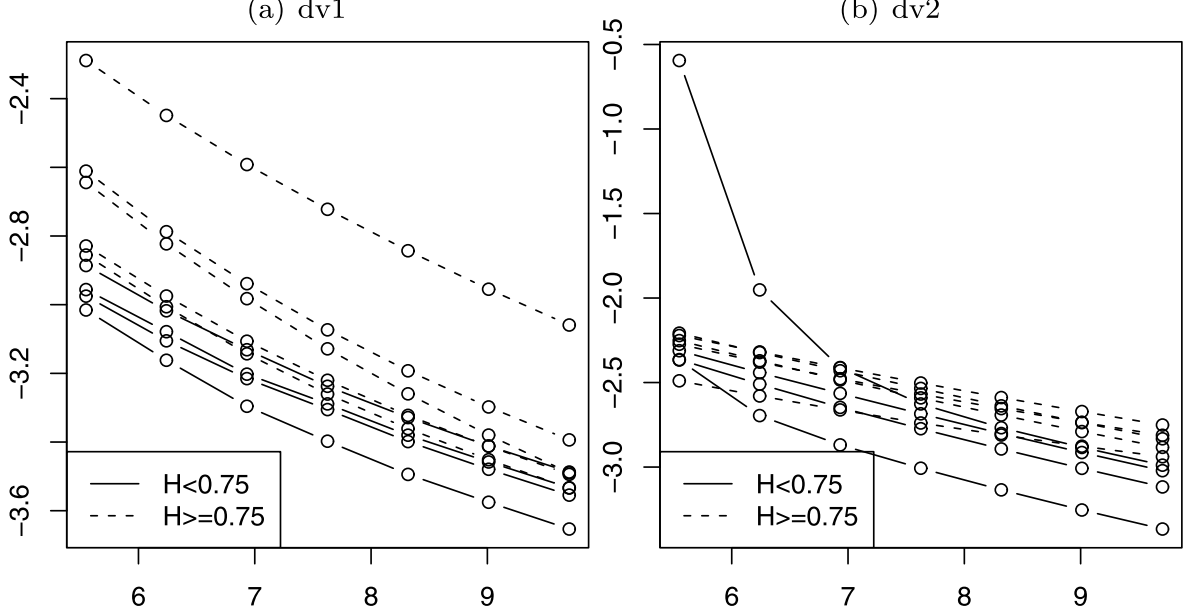

(c) osn

(d) oso
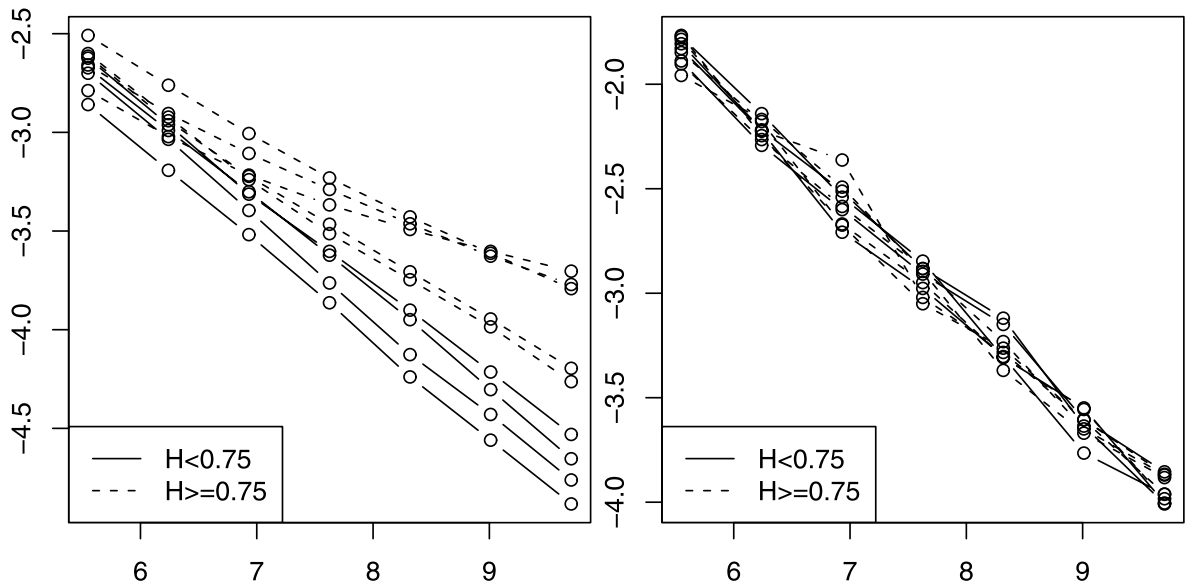

(e) $\operatorname{var}$

(f) ir

Fig. 6. Dependance of $\log (S D)$ against $\log (n)$ for the gBm process, $H \in\{0.55,0.6, \ldots, 0.95\}$. 
Table 4

Comparison of the estimators for the gBm process for sample path lengths $N=2^{k}+1$

\begin{tabular}{llrrrr}
\hline & $k$ & \multicolumn{1}{l}{10} & \multicolumn{1}{l}{12} & \multicolumn{1}{l}{14} \\
\hline MSE & dv1 & $\mathbf{0 . 0 5 2}$ & $\mathbf{0 . 0 2 8}$ & $\mathbf{0 . 0 1 6}$ & $\mathbf{0 . 0 0 7}$ \\
& dv2 & 0.103 & 0.065 & $\mathbf{0 . 0 2 8}$ & 0.013 \\
& var & 0.073 & $\mathbf{0 . 0 3 6}$ & $\mathbf{0 . 0 2 0}$ & $\mathbf{0 . 0 1 1}$ \\
gn & $\mathbf{0 . 0 6 6}$ & 0.050 & 0.041 & 0.034 \\
osn & 0.075 & 0.056 & 0.044 & 0.037 \\
go & 2.411 & 0.097 & 0.061 & 0.048 \\
oso & 0.556 & 0.090 & 0.063 & 0.050 \\
\hline ir & 0.172 & 0.079 & 0.037 & 0.018 \\
\hline dv1 & $-\mathbf{0 . 0 0 2}$ & $\mathbf{0 . 0 0 1}$ & $\mathbf{0 . 0 0 2}$ & $\mathbf{0 . 0 0 1}$ \\
dv2 & -0.020 & $-\mathbf{0 . 0 0 2}$ & $\mathbf{0 . 0 0 0}$ & $\mathbf{0 . 0 0 0}$ \\
var & -0.013 & $\mathbf{0 . 0 0 1}$ & $\mathbf{0 . 0 0 1}$ & $\mathbf{0 . 0 0 2}$ \\
gn & 0.093 & 0.074 & 0.062 & 0.054 \\
osn & 0.137 & 0.110 & 0.092 & 0.079 \\
go & 0.223 & -0.009 & -0.011 & -0.010 \\
oso & 0.065 & 0.006 & $\mathbf{0 . 0 0 1}$ & $\mathbf{0 . 0 0 0}$ \\
ir & -0.043 & -0.013 & -0.014 & -0.013 \\
\hline
\end{tabular}

(a) $H=0.65$

\begin{tabular}{lrrrr}
\hline$k$ & \multicolumn{1}{l}{10} & \multicolumn{1}{l}{12} & \multicolumn{1}{l}{14} \\
\hline dv1 & $\mathbf{0 . 0 4 7}$ & $\mathbf{0 . 0 3 3}$ & $\mathbf{0 . 0 2 3}$ & $\mathbf{0 . 0 1 6}$ \\
dv2 & 0.094 & $\mathbf{0 . 0 5 3}$ & $\mathbf{0 . 0 2 6}$ & $\mathbf{0 . 0 1 3}$ \\
var & 0.082 & $\mathbf{0 . 0 5 0}$ & $\mathbf{0 . 0 3 2}$ & 0.023 \\
gn & 0.082 & 0.059 & 0.046 & 0.037 \\
osn & 0.092 & 0.065 & 0.049 & 0.040 \\
go & 0.104 & 0.085 & 0.071 & 0.060 \\
oso & 0.109 & 0.090 & 0.075 & 0.064 \\
ir & 0.169 & 0.095 & 0.038 & 0.021 \\
\hline dv1 & $-\mathbf{0 . 0 0 5}$ & $-\mathbf{0 . 0 0 1}$ & $\mathbf{0 . 0 0 1}$ & $\mathbf{0 . 0 0 0}$ \\
dv2 & -0.016 & $\mathbf{0 . 0 0 3}$ & $-\mathbf{0 . 0 0 2}$ & $-\mathbf{0 . 0 0 1}$ \\
var & -0.025 & -0.007 & $-\mathbf{0 . 0 0 2}$ & $\mathbf{0 . 0 0 1}$ \\
gn & 0.094 & 0.075 & 0.062 & 0.054 \\
osn & 0.136 & 0.108 & 0.091 & 0.078 \\
go & -0.038 & -0.028 & -0.022 & -0.017 \\
oso & -0.011 & -0.009 & -0.006 & -0.004 \\
ir & -0.039 & 0.019 & 0.011 & 0.008 \\
\hline
\end{tabular}

(b) $H=0.85$

- The estimators $\widehat{H}_{\text {go }}$ and $\widehat{H}_{\text {oso }}$, the ordinary least squares versions of the previous estimators, display totally different behavior - their biases are comparable to those of the other estimators. However, their mean squared errors are considerably higher than those of other estimators and tend to decrease only slightly as the sample path length is increased. Additionally, both of these estimators require the sample path length to be equal to $2^{k}+1, k \in \mathbb{N}$, which means that, for sample paths of different length, some of the observations must be truncated.

- The estimators $\widehat{H}_{\mathrm{dv} 1}$ and $\widehat{H}_{\mathrm{var}}$ behaved differently for "small" and "large" values of $H$. As $H \in(1 / 2 ; 3 / 4)$, they displayed the best characteristics while for higher values of $H$ their performance was close to or worse than that of other estimators. $\widehat{H}_{\text {var }}$ displayed increased biases for shorter sample paths.

- The characteristics of $\widehat{H}_{\mathrm{dv} 2}$ were slightly worse than those of $\widehat{H}_{\mathrm{dv} 1}$ and $\widehat{H}_{\mathrm{var}}$ for shorter sample paths and $H<3 / 4$, and they were similar or better for longer sample paths and $H>3 / 4$. Additionally, this estimator showed no notable dependance of its behavior on the value of $H$. $\widehat{H}_{\text {ir }}$ displayed such a dependance only for rather long sample paths, but its biases and mean squared errors were higher. Having considered the linear regression $\log (S D) \sim \log (n)$ for these two estimators, the results suggest that for both these estimators $S D\left(\widehat{H}_{(\cdot)}\right) \sim \mathcal{O}\left(n^{-1 / 2}\right)$.

- Calculation times for the estimators $\widehat{H}_{\mathrm{dv} 1}, \widehat{H}_{\mathrm{dv} 2}$ and $\widehat{H}_{\mathrm{osn}}$ were about $0.02 s$ with 100 sample paths of the length $N=2^{8}+1$ and about $0.4 s$ with 100 sample paths 
of the length $N=2^{14}+1$. Calculation times of $\widehat{H}_{\text {gn }}$ were about twice lower and those of $\widehat{H}_{\text {go }}, \widehat{H}_{\text {oso }}$ and $\widehat{H}_{\text {ir }}$ were $2-5$ times higher.

As a conclusion, the results of this study suggest that when there's a reason to expect the Hurst index to be high or when the Hurst index is estimated from a sufficiently long sample path, the $\widehat{H}_{\mathrm{dv} 2}$ estimator performs best. If either of these assumptions is not present, then the $\widehat{H}_{\mathrm{dv} 1}$ and $\widehat{H}_{\mathrm{var}}$ would likely give a more precise estimate.

\section{References}

Bardet, J.-M. et al. (2003). Long-range dependence. In: Doukham, P. (Ed.), Theory and Applications of LongRange Dependence, Birkhäuser, Basel, pp. 579-625.

Begyn, A. (2005). Quadratic variations along irregular subdivisions for Gaussian processes. Electronic Journal of Probability, 10, 691-717.

Begyn, A. (2006). Generalized quadratic variations of gaussian processes: limit theorems and applications to fractional processes. $\mathrm{PhD}$ thesis.

Berzin, C., León, J. (2008). Estimation in models driven by fractional Brownian motion. Annales de l'Institut Henri Poincare (B) Probability and Statistics, 44(2), 191-213.

Coeurjolly, J.F. (2000). Simulation and identification of the fractional Brownian motion: a bibliographical and comparative study. Journal of Statistical Software, 50 (7).

Chronopoulou, A., Viens, F.G. (2010). Hurst index estimation for self-similar processes with long-memory. In: Recent Advances in Stochastic Dynamics and Stochastic Analysis (in press), World Scientific, Singapore.

Davis, G. (1996). Implicit image models in fractal image compression. In: Proceedings of the SPIE Conference on Wavelet Applications in Signal and Image Processing IV.

Dudley, R.M. (1999). Picard iteration and p-variation: the work of Lyons. In: Mini-Proceedings: Workshop on Product Integrals and Pathwise Integration, MaPhySto, Aarhus.

Gladyshev, E.G. (1963). A new limit theorem for stochastic processes with Gaussian increments. Theory Probability and its Applications, 6(1), 52-61.

Istas, J., Lang, G. (1997). Quadratic variations and estimation of the local Hölder index of a Gaussian process. Annales de l'Institut Henri Poincare (B) Probability and Statistics, 33, 407-436.

Kubilius, K. (2000). The existence and uniqueness of the solution of the integral equation driven by the fractional Brownian motion. Lithuanian Mathematical Journal, 40, 104-110.

Kubilius, K., Melichov, D. (2008). On estimation of the Hurst index of solutions of stochastic integral equations. Lithuanian Mathematical Journal, 48-49, 401-406.

Kubilius, K., Melichov, D. (2009). Estimating the Hurst index of the solution of a stochastic integral equation. Lithuanian Mathematical Journal50, 24-29.

Kubilius, K., Melichov, D. (2010). Quadratic variations and estimation of the Hurst index of the solution of SDE driven by a fractional Brownian motion. Lithuanian Mathematical Journal, 50(4), 401-417.

Li, M. (2010). Essay on teletraffic models (I). In: Proceedings of the 9th WSEAS International Conference on Applied Computer and Applied Computational Science, pp. 130-135.

Lyons, T. (1994). Differential equations driven by rough signals (I): an extension of an inequality of L.C. Young. Mathematical Research Letters, 1, 451-464.

Norvaiša, R., Salopek, D.M. (2002). Estimating the $p$-variation index of a sample function: an application to financial data set. Methodology and Computing in Applied Probability, 4(1), 27-53.

Nualart, D., Rǎşcanu, A. (2002). Differential equations driven by fractional Brownian motion. Collectanea Mathematica, 53 (1), 55-81.

R Development Core Team. (2009). R: A Language and Environment for Statistical Computing. http: / / www. R-project.org.

Wenlu, X., Weixin, X. (1997). Image object detection based on fractional Brownian motion. Journal of Electronics, 14(4), 289-294. 
K. Kubilius graduated from the Vilnius University in 1976. He received the $\mathrm{PhD}$ degree from Vilnius University (1981), the dr. habil. from the Vilnius University Institute of Mathematics and Informatics (1993). He was conferred the title of professor at Vilnius Gediminas Technical University in 2000. Currently, he is a senior researcher in the Stochastic Process Department in the Vilnius University Institute of Mathematics and Informatics. His research interests include stochastic processes, stochastic differential equations and their modeling, limit theorems.

D. Melichov graduated from the Vilnius Gediminas Technical University in 2007 receiving the master of science of statistics degree. He is a doctoral student at the Vilnius Gediminas Technical University. His $\mathrm{PhD}$ research topic is asymptotic properties and parameter estimation of the solutions of stochastic differential equations driven by the fractional Brownian motion.

\title{
Apie stochastinių diferencialiniu lygčiu, valdomu trupmeninio Brauno judesio, sprendiniu Hursto indekso ivertiniu palyginima
}

\author{
Kęstutis KUBILIUS, Dmitrij MELICHOV
}

Straipsnyje pateikiamas Hursto indekso įvertiniu tyrimas trupmeniniams Ornštein-Ulenbeck ir Black-Scholes modeliams. Nagrinejjama ịvertinių skaitinių charakteristikų priklausomybė tiek nuo Hursto indekso reikšmés, tiek nuo trajektorijos ilgio. 